Trieste

\title{
PER UN ETIMO DEL TOPONIMO CARSICO SLOV. OPČINA (ITAL. OPICINA)
}

Il nuovo etimo ${ }^{1}$ che qui propongo per il noto toponimo carsico, slov. Opčina, ital. Opicina (e ted. -nel passato-Obezenach, v. sotto), le cui prime attestazioni risalgono al 1308 e 1311 (forme documentate Obchena, Obchiena) ${ }^{2}$, mi è stato suggerito dalla lettura di un elenco di toponimi dalmati (croati) dell'isola di Uglian ed altre dell'arcipelago zaratino, elenco nel quale ho trovato un interessante Opatschina ${ }^{3}$, il quale differisce dal nostro Opčina unicamente per la presenza, dopo la labiale, di una $a$, vocale, questa, che nel toponimo isolano è certamente etimologica, in quanto appartiene alla base slava opât "abbate" (in subordine al noto opatija "abbazia"), vecchi adattamenti dalle voci tedesche ant. abt, apt, aptei (ted. mod. $A b t, A b t e i$ ), a sua volta risalenti a lat. (tardo) abbās, abbātia ecc. (cfr. Bezlaj e Skok ss.vv. opât, risp. òpat). Nell'ambito delle isole quarnerine e della Dalmazia insulare la forma Opatschina non è isolata e fa compagnia ad un abbastanza noto Opat, nome di una punta, colle (m.100) e insenatura dell'isola Incoronata ${ }^{4}$, ad un Opatija dell'isola di Veglia (glagolit. a. 1633, I. Jelenic JZ 6, 1966, p. 281) $)^{5}$ e ad un Opatova, punta all'entrata dello stretto di Catena, tra l'Isola Lunga e l'Incoronata (cfr. Segelhandbuch p. 341). Ad ogni modo, per quanto avremo da esporre a proposito del toponimo (carsico!) $O p(p) a c(c)$ hiasella, ancor più specifico appare il confronto con altra espressione toponimica insulare, questa volta dell' isola di Eso, Opatiae verth (=Vérh) (a. 1392, Hilje cit. p. 69) ${ }^{6}$, tale e quale l'Opachiaç del 1459 (Hilje p. 72) e l'Opačak Veli odierno (Scotti p. 136).

1 Per un elenco e discussione degli etimi precedenti v. R. FonTANOT, Pucinum-Opcina-Opicina, ATr N. S. 56 (1996) pp. 681-693 (in particolare 691 ss.)

2 -ena al posto di -ina (il noto suffisso slavo collettivizzante) è certo dialettale (cfr. Fontanot p. 689; il quale cita a confronto per Veglia la forma Dobregno, al posto di Dobrnj. Quanto all' $i$ che precede alle volte questo -ena, si tratterà, certamente, di un sussidio grafico per sottolineare il valore palatale di $c h$. a. 1453 (Hilje FOC 3, 1994, p. 55) "in Vgliano in loco vocato Opatschina".

4 Segelhandbuch p. 189. Il nome sarebbe stato, però, suggerito non dalla presenza di abati o abbazie ma dal fatto che l'altura presentava una stratificazione concentrica di terreni a simiglianza d'un berretto d'abate (cfr. Friganović, p. 61).

5 E si confronti il più noto Opatija (ital. Abbazia), spiaggia balneare e stazione climatica della costa liburnica a occidente di Fiume, con attestazioni a partire dalla fine del "500 ("Abbazia di S. Giacomo" o "della Preluca"). Un Opatija è attestato anche a Žonti, presso Portole, nelle vicinanze della quale troviamo anche un Opatica, ma in Istria queste forme risalenti ad opat sono disperse e non formano un nucleo cosi compatto come nell'arcipelago

6 Per Opathie verth val la pena di citare il passo che ci interessa per esteso: "In insula Ecii super terreno dicti zaratino. Si confronti, tuttavia ancora, un Opatovac presso Pedena per a. 1617-1626 (VHARP 1, 1971, p. 274). Monasterii Sancti Nicolai in loco uocato Opathie verth" (cfr. più avanti per l'a. 1397: "In insula Eçii in loco uocato Montis Abbatis": lo stesso luogo?). 
Questo toponimo dalmata si raccomanda in modo particolare per il fatto che esso deriva, più specificamente, da un vecchio aggettivo in -ja (ie. -yo), tratto dal sostant. apat, precisamente opatja-, il quale non ha nulla a che fare con opatija "abbazia" e si ripete, come già accennato, nel toponimo del Carso Goriziano (ora in Slovenia) slov. Opatja Sela, ital. Opacchiasella (variamente ortografato), da tradurre, evidentemente, "villaggio dell'abate"7 di cui conosciamo anche forme tedeschizzate tipo Appatzell (a. 1484: si noti la "restituzione" della $a$ - etimologica) e italianizzate tipo Oppochiasella (a. 1635) (nel ' 300 il villaggio veniva chiamato, più semplicemente, Sela). ${ }^{8}$

Fra i toponimi dalmati abbiamo lasciato, appositamente, fuori la località chiamata Opaticina dell'Isola Lunga (Hilje) ${ }^{9}$. Tale toponimo è solo apparentemente simile all'Opatschina sopra riferito, in quanto è, semplicemente, un derivato del sost. (cr.) opatica "monaca" o "badessa", quindi avente in comune solamente l'elemento di base opat, non il più specifico aggettivo *opatja-. Quindi morfologicamente, e anche concettualmente, diverso.

Ma ora torniamo a Opčina. L'identità, o quasi, con l'Opatchina dalmata e con l'Opača Selo carsico mi sembra fuori discussione, tale quindi da osar di proporre ${ }^{10}$ per esso il significato di "località o villaggio in cui esistevano delle proprietà facenti capo ad abati (si rammenti che -ina, da ie. -ōn-, è suffisso collettivizzante) o ad un'abbazia" del luogo (poi scomparsa, senza lasciar altre tracce) o di luogo più lontano, l'Opacchiasella stessa: per quest' ultima eventualità basterà ricordare il toponimo locale Devinčina (periferia di Prosecco), letter. "luogo dei possedimenti dei Conti di Duino (Devin)". Non occorre, poi, certo insistere che il nostro Opčina, così etimologizzato, si inserisce a pieno titolo nella nutrita serie dei toponimi sloveni tipo i ben noti Ajdovščina, Kozina, Planina, Markovščina ecc.

E ancora: una volta ricostruita per il nostro Opcina la forma etimologica *Opačina, diviene lecito, anche, sospettare che le forme, sporadicamente attestate, con vocale cosiddetta epentetica (di solito $e, i$ ) tipo Opechiena, Opichiena (dal 1596 in poi) ${ }^{11}$ siano non secondarie, ma originali, relitti di una fase in cui coesistevano, dialettalmente, ambedue le forme, quelle senza sincope della $-a^{-}{ }^{12}$ e quelle, ancor più vicine al modello tedesco ant. tipo apt, aptei, con una sincope riconducibile al fatto ben noto che in tutti i momenti della sua storia lo sloveno (e dialetti sloveni) risentì più o meno l'influsso tedesco. E questo per Opčina lo possiamo affermare a ragion veduta, in quanto

7 Il Baroncelli (p. 6), difatti, italianizza bene quando propone un suo "Villabate".

8 Su Opača Sela v. riassuntivamente Bezlaj s.v. opat.

9 "in loco uocato Opaticina draga" (Hilje p. 69).

10 Il prezioso suggerimento mi è stato dato dal mio collega e amico Franco Crevatin, che qui ringrazio vivamente.

11 Per queste forme si rimanda nuovamente a Fontanot, p. 687, il quale non crede che la forma italianeggiante moderna Opicina riprenda le forme più antiche tipo Opechina ecc., come a dire che esse sarebbero sorte indipendentemente, onde evitare il contatto, accettabile per lo sloveno ma intollerabile per l'italiano, di $p$. e $\check{c}$.

12 Comunque già passata in atonia. 
in un'altra zona di dialetti slavi (precisamente ceki), dove il tedesco esercitò la sua pressione, ci è dato trovare all'interno di questa stessa famiglia di parole, una forma come jeptiška "badessa", evidentemente da ted. ant. eppetisse "id.", ma con una sincope identica a quella che abbiamo or ora riscontrato per Opčina.

E qui potremmo anche terminare il nostro discorso. Sennonché mi sento obbligato di dire ancora qualcosa a proposito di alcune forme relative al nostro toponimo. Abbastanza facile, ora, spiegare, la labiale sonora delle attestazioni più arcaiche tipo Obche-, Obchiena: di nuovo, qui, sarà responsabile l'influsso tedesco, con le sue forme tipo Abt, Abtei ortografate con la $b$ anziché con la $p$.

Più misteriose le note forme agg.-etn. Openci, Openski. Direi che alla luce della nuova etimologia esse non offrono particolari difficoltà: saranno, semplicemente, delle 'Kurzformen', partenti da un più completo Opačinski, sostenuto dall'esistenza di un (cr.) opatenski, effettivamente attestato per l'a. 1566 (Skok cit.) e formato alla stessa maniera.

$\mathrm{ATr}$ "Archeografo Triestino", Trieste

Baroncelli E. Baroncelli, Repertorio geografico per la guerra mondiale, I. Venezia Giulia e Dalmazia, Firenze 1915.

Bezlaj $\quad$ F. Bezlaj, Etimološki slovar slovenskega jezika, II, Ljubljana 1986.

FOC Folia Onomastica Croatica, Zagreb

Fontanot v. n. 1 .

Friganović $\quad$ M. Friganović, Parco nazionale Arcipelago Kornati, Zagabria 1990.

Hilje $\quad$ E. Hilje, Prilog povjesnoj topografiji zadarskog otočja, FOC 3 (1994), pp. 49-76.

$\mathrm{JZ}$ Jadranski Zbornik, Rijeka

Scotti G. Scotti, Gli arcipelaghi di Zara e Sebenico, Milano 1984.

Segelhandbuch Segelhandbuch der Adria, Pola 1906 (a cura della k. k. Kriegsmarine).

Skok P. Skok, Etimologijski riječnik hrvatskoga ili srpskoga jezika, II, Zagreb 1972.

VHARP Vijesnik Historijskih Arhiva u Rijeci i Pazinu, Rijeka.

Povzetek

K IZVORU KRAŠKEGA TOPONIMA SLOV. OPČINA (ITAL. OPICINA)

Kraški toponim Opčina je izveden iz slov. *Opačina, "kraj, kjer biva opat (ali kjer je opatija)", kar je izpričano kot Opatschina tudi v hrvaški toponomastiki iz 15. stoletja na otoku Ugljenu (Zadar). $Z a$ premik naglasa $z$ naglašenega $-a$ - in njegovo poznejše izginotje naj bi bil nekako odgovoren splošni vpliv nemščine. Tak razvoj dokazujejo vmesne oblike kot Opachiena (s konca 16. stoletja).. 